Article

\title{
Exosomes of Epstein-Barr Virus-Associated Gastric Carcinoma Suppress Dendritic Cell Maturation
}

\author{
Munetoshi Hinata ${ }^{1}$, Akiko Kunita ${ }^{1}$, Hiroyuki Abe ${ }^{1}$, Yasuyuki Morishita ${ }^{1}$, Kei Sakuma ${ }^{1}$, \\ Hiroharu Yamashita ${ }^{2}$, Yasuyuki Seto ${ }^{2}$, Tetsuo Ushiku ${ }^{1}$ and Masashi Fukayama ${ }^{1,3, *(D)}$ \\ 1 Department of Pathology, Graduate School of Medicine, The University of Tokyo, Tokyo 113-0033, Japan; \\ fircothep262@gmail.com (M.H.); kunita@m.u-tokyo.ac.jp (A.K.); hiroyuki.abe.1101@gmail.com (H.A.); \\ moriyasu@m.u-tokyo.ac.jp (Y.M.); k-sakuma@m.u-tokyo.ac.jp (K.S.); usikut@gmail.com (T.U.) \\ 2 Department of Gastrointestinal Surgery, Graduate School of Medicine, The University of Tokyo, \\ Tokyo 113-0033, Japan; hiroharu.yamashita@gmail.com (H.Y.); seto-tky@umin.ac.jp (Y.S.) \\ 3 Asahi TelePathology Center, Asahi General Hospital, Asahi City, Chiba 289-2511, Japan \\ * Correspondence: mfukayama-tky@umin.org; Tel.: +81-3-5841-3343
}

Received: 10 October 2020; Accepted: 10 November 2020; Published: 12 November 2020

\begin{abstract}
The Epstein-Barr virus (EBV)-associated gastric carcinoma (EBVaGC) is characterized by the infiltration of lymphocytes and a unique tumor microenvironment. Exosomes from cancer cells are essential for intercellular communication. The aims of this study were to investigate the secretion of EBVaGC exosomes and their physiological effect on dendritic cell maturation in vitro and to characterize dendritic cells (DCs) in EBVaGC in vivo. Western blotting analysis of CD63 and CD81 of exosomes from EBV-infected gastric cancer cell lines indicated an increase in exosome secretion. The fraction of monocyte-derived DCs positive for the maturation marker CD86 was significantly suppressed when incubated with exosomes from EBV-infected gastric cancer cell lines. Immunohistochemical analysis of GC tissues expressing DC markers (S100, Langerin, CD1a, CD83, CD86, and BDCA-2) indicated that the density of DCs was generally higher in EBVaGC than in EBV-negative GC, although the numbers of CD83- and CD86-positive DCs were decreased in the group with high numbers of CD1a-positive DCs. A low number of CD83-positive DCs was marginally correlated with worse prognosis of EBVaGC in patients. EBVaGC is a tumor with abundant DCs, including immature and mature DCs. Moreover, the maturation of DCs is suppressed by exosomes from EBV-infected epithelial cells.
\end{abstract}

Keywords: Epstein-Barr virus; gastric cancer; exosome; dendritic cells

\section{Introduction}

The Epstein-Barr virus (EBV) is a $\gamma$-herpesvirus and has been associated with neoplastic growth of various human cells, including lymphocytes of B-, T-, and NK-cell lineages and epithelial cells of the nasopharynx and stomach [1]. How EBV initiates and maintains neoplastic growth of latently infected cells that counteract immune surveillance in a specific milieu of each cell type has not yet been elucidated. EBV-associated gastric carcinoma (EBVaGC) is characterized by the clonal growth of EBV-infected stomach epithelial cells, and the frequency of EBVaGC takes up nearly $10 \%$ of gastric carcinoma cases [2]. Many cases of gastric carcinoma are treated by endoscopy and surgery; however, 5 -year survival rates still range from $30 \%$ to $70 \%$ [3]. EBVaGC has a unique histologic subtype that is known as gastric carcinoma with lymphoid stroma (GCLS); EBVaGC requires intensive investigation such that its interaction with immune cells, especially in this type of EBV-infected malignant tumor, is elucidated. 
The tumor microenvironment, which consists of inflammatory cells, stromal cells, vasculatures, and extracellular matrix, is an indispensable factor in the pathophysiology of cancers. Inflammatory cells include lymphocytes macrophages, neutrophils, and dendritic cells (DCs) [4]. During the development of the tumor microenvironment, intercellular communications are accomplished through the secretion of proteins and nucleotides and the various forms of cell-to-cell contact such as ligand-receptor interactions on the cell surface. Recently, exosomes or extracellular vesicles from cancer cells were reported as important tools for communication [5]. The elucidation of the characteristics of exosomes and their role in tumor development is expected to contribute on how the tumor microenvironment and cancer growth can be manipulated and suppressed.

Pegtel et al. [6] demonstrated that EBV-encoded microRNAs (miRNAs) are secreted by EBV-infected B cells through exosomes. EBV-miRNAs are internalized by non-infected dendritic cells, thereby downregulating immunoregulatory genes. Dendritic cells, which conduct acquired immunity, are also abundant in EBVaGC; however, their significance has not yet been fully elucidated [7-10]. The aims of this study were to (1) characterize the physical properties of EBVaGC exosomes, (2) identify their physiological effects on dendritic cell maturation in vitro, and (3) evaluate dendritic cells in EBVaGC in vivo. In the present study, we employed the use of dendritic cell markers suitable for immunohistochemical analysis as previously reported [11] to visualize tumor-associated dendritic cells (TA-DCs) and bridge findings from the flow cytometry analysis of gastric cancer-derived exosomes with those of the immunohistochemical analysis of gastric cancer tissues; the dendritic cell markers we used include CD1a, which is a marker for immature monocyte-derived DCs (moDCs), CD83 and CD86 for mature or activated DCs [12], and other markers (S100, Langerin, and BDCA-2). The maturation of moDCs was considerably suppressed in EBVaGC, which might have been mediated through the excretion of exosomes from the neoplastic cells infected with EBV.

\section{Materials and Methods}

\subsection{Culture of Gastric Cancer Cell Lines}

For in vitro experiments, gastric cancer cell lines MKN7, MKN74 (Riken BioResource Center Cell Bank, Tsukuba, Japan), and SNU719 (Korean Cell Line Bank, Seoul, Korea) were used. MKN7 and MKN74 were originally derived from well and moderately differentiated gastric adenocarcinoma without EBV infection, respectively. SNU719 was derived from EBVaGC. MKN7 and MKN74 were artificially infected with neomycin-resistant recombinant EBV in our laboratory, according to a cell-to-cell contact method [13] to yield stable infected gastric cancer cell lines MKN7 + EBV and MKN74 + EBV, respectively. These cell lines were cultured in RPMI 1640 medium (Nacalai Tesque, Kyoto, Japan) supplemented with 10\% fetal bovine serum (CELLect; MP Biomedicals, Irvine, CA, USA) and penicillin (100 U/mL)-streptomycin $(100 \mu \mathrm{g} / \mathrm{mL})$ mixed solution and incubated at $37^{\circ} \mathrm{C}$ in a humidified atmosphere containing $5 \% \mathrm{CO}_{2}$. For the culture of MKN7 + EBV and MKN74 + EBV, $50 \mathrm{\mu g} / \mathrm{mL}$ G-418 Solution (Roche Diagnostics, Basel, Switzerland) was also added to the culture medium.

The Burkitt lymphoma cell line Akata, used in the cell-to-cell contact method, was a gift from Prof. Kenzo Takada (HokkaidoUnivesity). Namalwa (CRL-1432) and Raji (CCL-86) were also Burkitt lymphoma cell lines, and used as a control for evaluation of EBV-DNA and latent and lytic genes of EBV. Both cell lines were purchased from American Type Culture Collection (Manassas, VA, USA). These cells were similarly cultured as described above.

\subsection{RNA Isolation and RT-PCR}

Total RNA was extracted from gastric cancer cells using ISOGENII (Nippon Gene, Tokyo, Japan). Total RNA (0.5 $\mathrm{\mu g}$ ) was used for cDNA synthesis with ReverTra Ace qPCR RT Kit (Toyobo, Osaka, Japan). The mRNA levels were measured by Thermal Cycler Dice Real Time System (TaKaRa, Shiga, Japan) and KAPA SYBR Fast qPCR Kit (Kapa Biosystems, Wilmington, MA, USA). The primer sequences were as follows; EBNA1-Fwd: 5'-CCTCCCTGGTTTCCACCTAT-3', 
EBNA1-Rev: 5'-TCCTCACCCTCATCTCCATC-3', BZLF1-Fwd: 5'-CTGGTGTCCGGGGGATAAT-3', BZLF1-Rev: 5'-TCCGCAGGTGGCTGCT-3', GAPDH-Fwd: 5'-CAACGGATTTGGTCGTATTGG-3', and GAPDH-Rev: 5'-GCAACAATATCCACTTTACCAGAGTTAA-3'.

\subsection{Exosome Isolation and Purification}

Exosomes were collected according to the method described by Yoshioka et al. [14] In brief, gastric cancer cell lines were washed with phosphate-buffered saline (PBS) twice and cultured in growth media (Advanced RPMI1640, Thermo Fisher Scientific, Waltham, MA, USA) without serum for $48 \mathrm{~h}$, followed by the collection of the culture supernatant. There was no additional procedure to induce exosome production. Most cells were viable after the $48 \mathrm{~h}$ culture, and the total cell number was about $1 \times 10^{8}$ cells for each cell line. Afterwards, $200 \mathrm{~mL}$ supernatant solutions from each cell line culture was centrifuged at $300 \times g$ for $10 \mathrm{~min}$, and the resulting supernatant was subsequently centrifuged at $9240 \times g$ for $40 \mathrm{~min}$. Then, the supernatant was filtered through a $0.22-\mu \mathrm{m}$ cellulose acetate membrane filter (Corning, New York, NY, USA), and cell debris was thoroughly removed. The filtrate was concentrated by ultrafiltration using Amicon Ultra 15 Ultracel $50 \mathrm{kDa}$ (Merck Millipore, Burlington, MA, USA), and 10-30 mL of samples was obtained. These samples were ultracentrifuged at $110,000 \times g$ for $70 \mathrm{~min}$, and the supernatant was discarded. Then, the samples were redissolved in PBS and ultracentrifuged at $110,000 \times g$ for 70 min again.

\subsection{Preparation of Whole Cell Lysates}

Cultured cell lines (about $1 \times 10^{7}$ cells for each cell line) were washed twice with PBS, and $500 \mu \mathrm{L}$ lysis buffer was added to each culture plate that was placed on ice. Thereafter, the cells were scraped with a cell scraper and mixed by pipetting and vortexing. The mixture was allowed to stand for $30 \mathrm{~min}$ and centrifuged at 15,000 rpm for $30 \mathrm{~min}$; the resulting supernatant was collected. For the preparation of lysates under non-reducing conditions, a lysis buffer comprising $50 \mathrm{mM}$ Tris- $\mathrm{HCl} \mathrm{pH} \mathrm{7.5,}$ $150 \mathrm{mM} \mathrm{NaCl}$, and $1.0 \% \mathrm{NP}-40$ was used. For the preparation of lysates under reducing conditions, RIPA buffer comprising $50 \mathrm{mM}$ Tris- $\mathrm{HCl} \mathrm{pH} 7.5,150 \mathrm{mM} \mathrm{NaCl}, 1 \%$ Triton X-100, 0.1\% sodium dodecyl sulfate (SDS), $10 \%$ glycerol, $2 \mathrm{mM}$ EDTA, and $10 \mathrm{mM} \mathrm{NaF}$ was used.

\subsection{Western Blotting}

Western blotting of exosomes was performed according to the recommendation (MISEV2018) [15]. Exosome samples (equivalent to the amount of exosome in $5 \times 10^{5}$ cells per each sample) or whole cell lysates (20 $\mu \mathrm{g}$ of protein per each sample) were incubated with $6 \mathrm{X}$ sample buffer ( $350 \mathrm{mM}$ Tris- $\mathrm{HCl}$ $\mathrm{pH} 6.8,10 \% \mathrm{SDS}, 30 \%$ glycerol, at $37^{\circ} \mathrm{C}$ for $30 \mathrm{~min}$ for preparation under non-reducing conditions; $350 \mathrm{mM}$ Tris- $\mathrm{HCl} \mathrm{pH}$ 6.8, 10\% SDS, $500 \mathrm{mM}$ dithiothreitol [DTT], 30\% glycerol, at $95{ }^{\circ} \mathrm{C}$ for $5 \mathrm{~min}$ for preparation under reducing conditions). The samples were transferred onto a polyvinylidene fluoride (PVDF) membrane and blocked with tris-buffered saline (TBS) with Tween 20 containing $5 \%$ skim milk. The samples were incubated with primary antibodies at $25 \pm 2{ }^{\circ} \mathrm{C}$ for $2 \mathrm{~h}$. Then, the samples were washed and incubated with HRP-conjugated secondary antibody at $25 \pm 2{ }^{\circ} \mathrm{C}$ for $1 \mathrm{~h}$. The samples were washed, and chemiluminescence signals were detected using a chemiluminescence kit (ImmunoStar Reagent or ImmunoStar LD, FUJIFILM Wako Pure Chemical Corporation, Osaka, Japan). Anti-CD63 (BD Pharmingen, San Diego, CA, USA, clone H5C6, 1:200), anti-CD81 (Santa Cruz Biotechnology, Santa Cruz, CA, USA, clone 5A6, 1:200), and anti- $\beta$-actin (A5441; Sigma-Aldrich, St. Louis, MO, USA, 1:10,000) antibodies were used as primary antibodies. Procedures were performed under non-reducing conditions for CD63 and CD81, and under reducing conditions for $\beta$-actin.

\subsection{Transmission Electron Microscopy}

A formvar membrane was placed on HF36 400 mesh (Pyser-SGI, Edenbridge, Physer-SGI, UK), dried, and carbon-deposited. The exosome sample was placed thereon, excess liquid was removed with filter paper after $2-3 \mathrm{~s}$. Then, a small drop of $1 \%$ uranium acetate was applied for a few seconds; 
the membrane was blotted with filter paper and air-dried. The samples were observed with JEM-1400 Transmission Electron Microscope (JEOL, Tokyo, Japan).

\subsection{Culture and Stimulation of Dendritic Cells Derived from Human Monocytes}

Peripheral blood was collected from healthy donors. Blood fractions containing mononuclear cells were separated using Ficoll Paque Plus (Cytiva, Marlborough, MA, USA) according to the manufacturer's instructions. After the blood was centrifuged at $300 \times g$ for $10 \mathrm{~min}$ at $25 \pm 2{ }^{\circ} \mathrm{C}$, the sediment was suspended in $80 \mu \mathrm{L}$ autoMACS running buffer (Miltenyi Biotec, Bergisch Gladbach, Germany) per $1 \times 10^{7}$ cells. Then, $20 \mu \mathrm{L}$ CD14 MicroBeads (Miltenyi Biotec) per $1 \times 10^{7}$ cells was added and incubated at $4{ }^{\circ} \mathrm{C}$ for $15 \mathrm{~min}$. Afterwards, $1-2 \mathrm{~mL}$ buffer was added per $1 \times 10^{7}$ cells, and the mixture was centrifuged at $4{ }^{\circ} \mathrm{C}, 300 \times \mathrm{g}$ for $10 \mathrm{~min}$, and the supernatant was discarded. The sediment was suspended in $500 \mu \mathrm{L}$ buffer. Thereafter, CD14-positive monocytes were collected using a magnetic cell separator according to the manufacturer's instructions. An aliquot was used for fluorescence-activated cell sorting (FACS), and the rest of the sample was transferred on a 6-well plate at a density of $1.5 \times 10^{6}$ cells $/ 5 \mathrm{~mL} /$ well and cultured at $37^{\circ} \mathrm{C}$ in a humidified atmosphere containing $5 \% \mathrm{CO}_{2}$; the culture medium used comprised RPMI 1640, 10\% FBS, $1 \%$ penicillin-streptomycin mixed solution, IL-4 (Wako, $50 \mathrm{ng} / \mathrm{mL}$ ), and GM-CSF (Wako, $50 \mathrm{ng} / \mathrm{mL}$ ). Monocytes incubated in a medium without IL-4 and GM-CSF, which are used for dendritic cell differentiation, were used as control.

Exosome samples derived from the gastric cancer cell line were diluted to $20 \mu \mathrm{g} / \mathrm{mL}$ (protein concentration measured by using bicinchoninic acid (BCA) assay) in PBS and sterilized by filtration with $0.22 \mu \mathrm{m}$ sterile filter (MILLEX-GV, Merck Millipore, Cork, Ireland). Subsequently, $2 \mu \mathrm{g}$ exosomes were added to the culture on days 0,3 , and 5; on the other hand, $100 \mu \mathrm{L}$ PBS, instead of exosomes, was added to separate cultures as controls that simulate immature DC. Furthermore, onto the cultures as a control that simulates mature dendritic cells, incubation, addition of $100 \mu \mathrm{L}$ PBS on days 0,3 , and 5 , and addition of $1 \mu \mathrm{g} / \mathrm{mL}$ lipopolysaccharides (Sigma-Aldrich) on day 5 were performed.

Six days after the start of the culture, the cells were detached by pipetting and scraping and were collected. After centrifugation at $1500 \mathrm{RPM}$ for $10 \mathrm{~min}$ at $25 \pm 2{ }^{\circ} \mathrm{C}$, the supernatant was discarded, and the sediment resuspended in $205 \mu \mathrm{L}$ PBS. The number of cells was counted and aliquoted into $50 \mu \mathrm{L}$ solutions, to which $2.5 \mu \mathrm{L}$ primary antibody was added; the solutions were incubated on ice for $15 \mathrm{~min}$. Then, $500 \mu \mathrm{L}$ PBS was added, and the mixture was centrifuged at $2000 \mathrm{RPM}$ at $4{ }^{\circ} \mathrm{C}$ for $10 \mathrm{~min}$. The supernatant was removed, and the sediment resuspended in $500 \mathrm{~mL}$ of PBS. This was used for analysis by FACS. The primary antibodies used were CD1a-PE (BioLegend, San Diego, CA, USA, clone HI149) and CD86-PE (TONBO Biosciences, San Diego, CA, USA, clone IT2.2).

\subsection{Tissue Samples}

Pathological records were reviewed, and formalin-fixed paraffin-embedded gastric cancer tissues were obtained from the archives of the Department of Pathology, The University of Tokyo Hospital. EBVaGC cases were collected, and EBV-negative gastric cancer cases were also included as control samples. EBV infection in cancer cells was confirmed by EBER-in situ hybridization. For immunohistochemical staining, tissue microarray data constructed from these cases were used. The study was conducted in accordance with the Declaration of Helsinki, and the protocol was approved by the Ethics Committee of the Graduate School of Medicine, the University of Tokyo (10461-(10)).

\subsection{Immunohistochemistry}

Immunohistochemical staining for Langerin, CD1a, S100, CD83, CD86, and BDCA-2 was performed on tissue microarray using VENTANA BenchMark, an automated immunostainer (Roche Diagnostics). The staining conditions were as follows: CC1 buffer for $60 \mathrm{~min}$, primary antibody reaction for $32 \mathrm{~min}$, and visualization with iVIEW DAB v3. The primary antibodies used were mouse monoclonal anti-Langerin antibody (Leica Biosystems, Wetzlar, Germany, clone 12D6, 1:200), mouse monoclonal anti-CD1a antibody (Agilent Technologies, Santa Clara, CA, USA, clone O10, 1:100), 
rabbit polyclonal anti-S100 antibody (Roche Diagnostics), mouse monoclonal anti-CD83 antibody (Santa Cruz Biotechnology, clone Hb15a, 1:100), rabbit monoclonal anti-CD86 antibody (Cell Signaling Technology, Danvers, MA, USA, clone E2G8P, 1:50), and mouse monoclonal anti-BDCA-2 antibody (DENDRITICS, Lyon, France, clone 124B3.13, 1:50).

To evaluate the number of immune cells, two tissue microarray cores were used in each case of gastric carcinoma. Each core comprised approximately $4 \mathrm{~mm}^{2}$ tissue; therefore, a total of $8 \mathrm{~mm}^{2}$ tissue area was evaluated for each case. Tissue slides were digitized using a Nanozoomer 2.0-HT virtual slide scanner (Hamamatsu Photonics, Hamamatsu, Japan), and tumor areas were measured using NDP.view2 software (Hamamatsu Photonics). The area containing viable tumor cells was considered the tumor area. All tumor areas were specified and measured for each tissue microarray core. The ratio of tumor area to whole tissue area differed from case to case (from $5 \%$ to $100 \%$ ). The dendritic cells expressing each marker (Langerin, CD1a, S100, CD83, CD86, and BDCA-2) that infiltrated the tumor areas were visually counted under an optical microscope (ECLIPSE 80i, Nikon, Tokyo, Japan). As some dendritic cells have a morphologically complex cytoplasm, a cell whose nucleus was recognizable was counted as a distinct cell. Among S100-positive cells, those considered to be morphologically macrophages were excluded. The density of DCs was calculated by dividing the cell number by the area of the tumor.

\subsection{Analysis of Correlation between the Density of Dendritic Cells and Clinicopathological Characteristics}

Gastric cancer cases were divided into subgroups according to clinicopathological parameters, including age, sex, tumor size, Lauren classification, tumor depth, venous invasion, lymphatic invasion, and lymph node metastasis. The mean value of the density of DCs was calculated for each subgroup. Gastric cancer cases were also divided into two groups according to the density of DCs that expressed each marker, and overall survival was compared using the Kaplan-Meier method. As a threshold of DC density, the median value in each subgroup of EBVaGC and EBV-negative gastric cancer, respectively, was used, as DC density differed greatly between EBVaGC and EBV-negative gastric cancer.

\subsection{Statistical Analysis}

For the comparison of DC density that infiltrated gastric cancer tissue and the comparison of exosome size on transmission electron microscopy, Wilcoxon rank sum test was used. For the comparison of overall survival, log-rank test was used. Statistical analysis was performed using R software (version 3.2.3; R Foundation for Statistical Computing, Vienna, Austria) [16].

\section{Results}

\subsection{Exosomes Purified from Gastric Cancer Cell Lines with and without EBV Infection}

To evaluate the exosomes of gastric cancer cell lines with EBV-infection (MKN7 + EBV and MKN7 + EBV) and of a cancer cell lines from an EBV gastric carcinoma (SNU719), the presence of EBV was confirmed by positive results of EBNA1-RT-PCR and the absence of lytic infection by negative results of BZLF1-RT-PCR, respectively (Figure 1A). Then, exosomes were extracted from the culture media of MKN7, MKN7 + EBV, MKN74, MKN7 + EBV, and SNU719 by ultracentrifugation.

CD63 and CD81, which are exosome marker proteins, were evaluated in cell lysates and exosome samples from gastric cancer cell lines (Figure 1). Increased levels of CD63 and CD81 were observed in both cell lysates and exosome samples in MKN7 + EBV and MKN74 + EBV, respectively, compared to those in MKN7 and MKN74. The amount of CD63 and CD81 were much greater in SNU719 than in MKN7 + EBV and MKN74 + EBV.

The integrity of purified exosomes was confirmed by transmission electron microscopy as small vesicles with 30-100 $\mu \mathrm{m}$ diameter (Figure 2A-C) were observed. The diameters of exosomes from MKN74, MKN74 + EBV, and SNU719 were $71.3 \pm 43.7 \mathrm{~nm}(n=6), 33.9 \pm 8.5 \mathrm{~nm}(n=25)$, 
and $69.6 \pm 42.9 \mathrm{~nm}(n=29)$, respectively. The diameter of the exosomes in MKN74 + EBV was significantly smaller than that in MKN74 ( $p<0.0001$, determined by using Wilcoxon rank sum test).
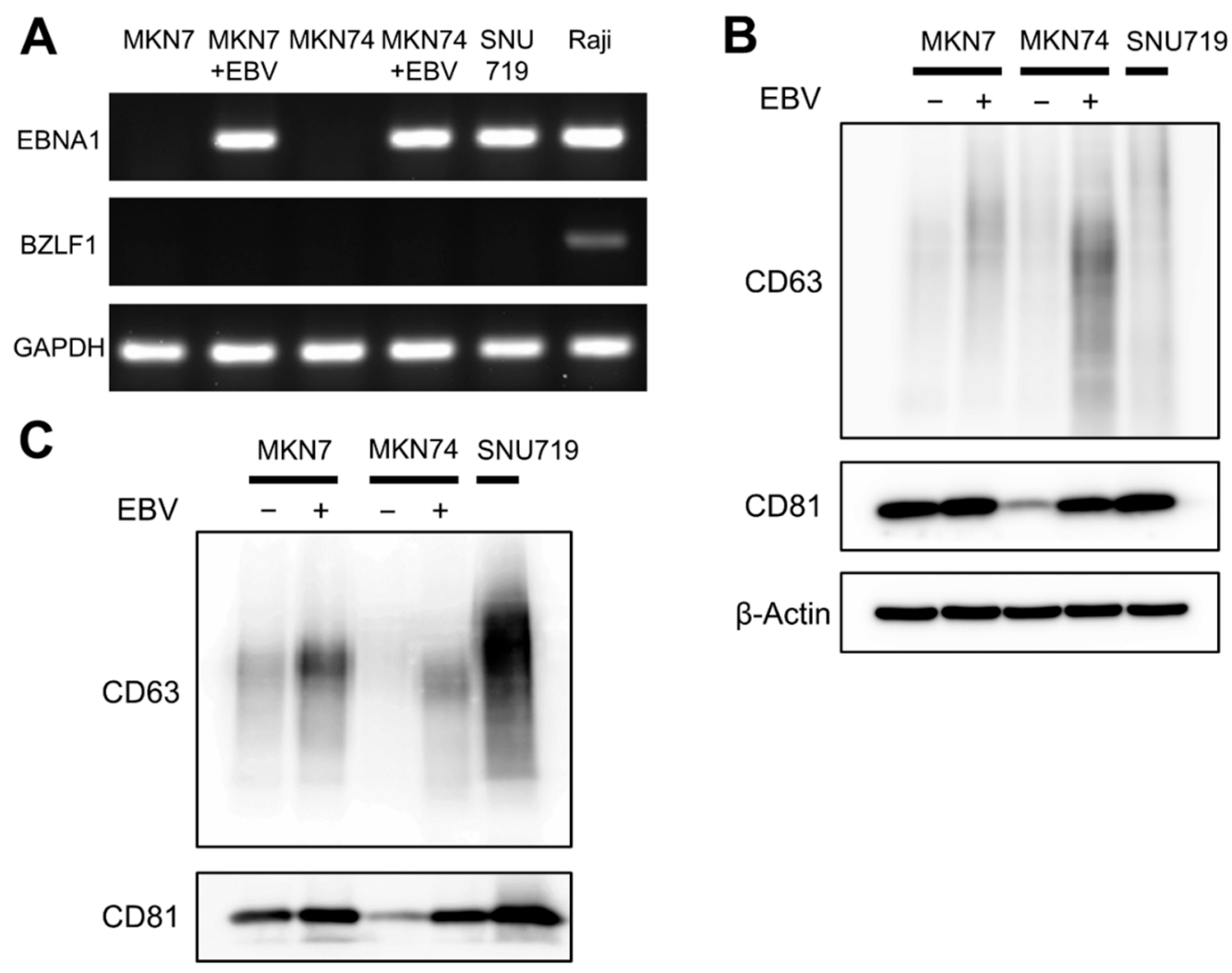

Figure 1. Detection of exosome marker proteins in gastric cancer cell lines with and without EBV-infection (MKN7, MKN7 + EBV, MKN74, and MKN74 + EBV) and the gastric cancer cell line of EBVaGC (SNU719). (A): PCR evaluation of an EBV-latent gene, EBNA1 and RT-PCR evaluation of a lytic gene, BZLF1. (B,C): Western blot analysis of exosome marker proteins in cell lysates (B) and exosomes $(\mathbf{C})$. Proteins from the same amount of cell lysate $(20 \mu \mathrm{g}$ of protein per each sample) or exosomal preparations from the same number of cells from gastric cancer cell lines (equivalent to the amount of exosome in $5 \times 10^{5}$ cells per each sample) were separated by SDS-PAGE, transferred onto a PVDF membrane, and incubated with specific antibodies (CD63, CD81, $\beta$-Actin). Signals were detected with HRP-conjugated secondary antibody and by using a chemiluminescence kit.

A

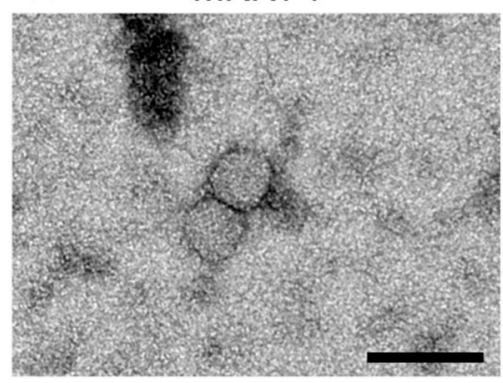

B MKN74+EBV

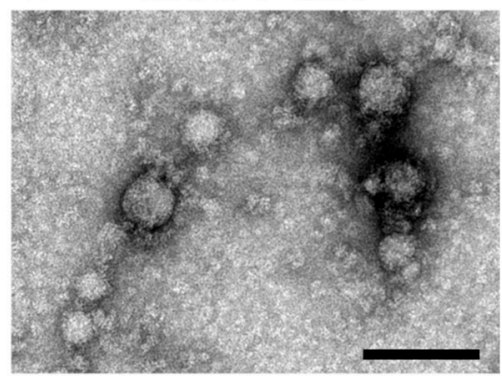

C $\quad$ SNU719

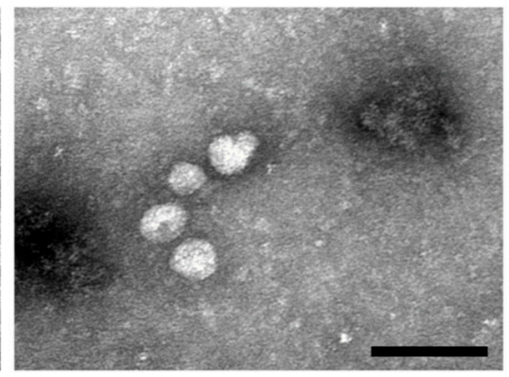

Figure 2. Characterization of exosomes by transmission electron microscopy (A-C). Exosomes were extracted from culture supernatant of gastric cancer cell lines by ultracentrifugation. Exosomes were recognized as small vesicles 30-100 nm in diameter under transmission electron microscopy. (A) MKN74, (B) MKN74 + EBV, (C) SNU719. Scale bar: $100 \mathrm{~nm}$. 


\subsection{Effect of Artificial EBV-Infection on Exosomes of Gastric Cancer Cells}

To evaluate the effect of artificial EBV infection, western blotting and transmission electron microscopy were performed (Table 1). The effects of EBV infection on exosomes were almost similar with those on cell lysates. We assume a simple model as follows: (1) exosomes are spheres, (2) protein amounts are proportional to the particle volume, and (3) the densities of CD63 and CD81 on the particle surface are constant. Thus, the densities of CD63 and CD81 are proportional to the surface area of the particle surface. Since the diameter of MKN74 + EBV exosomes was nearly half of MKN74 exosomes, the number of particles was calculated as 8-fold and the protein content of each particle 0.125-fold (Appendix A).

Table 1. Effect of artificial EBV-infection.

\begin{tabular}{cccc}
\hline & Protein $(\mu \mathrm{g} / \mathbf{1 0 5}$ Cell) Fold & CD63 Density Fold & CD81 Density Fold \\
\hline Cell lysate & & & \\
\hline MKN7 + EBV/MKN7 & & 2.147 & 2.973 \\
\hline MKN74 + EBV/MKN74 & & & \\
\hline Exosome & $0.505 / 0.515$ & 2.046 & 1.601 \\
\hline MKN7 + EBV/MKN7 & 0.981 & 2.104 & not applicable \\
\hline MKN74 + EBV/MKN74 & $0.310 / 0.380$ & & 2.878 \\
\hline
\end{tabular}

Total protein concentration of exosome sample was measured by BCA assay and adjusted against the original cell number. CD63 and CD81 density was obtained by the densitometry of Western blots. The size of exosomes was obtained by electron microscopy. Not applicable: electron microscopy was not performed for MKN7 or MKN7 + EBV.

\subsection{Exosomes of EBVaGC Gastric Cancer Cells}

The results of the evaluation of exosomes in SNU719 by Western blotting and transmission microscopy were compared to those of EBV-non-infected gastric cancer cell lines MKN7 and MKN74 (Table 2). The densities of CD63 and CD81 were nearly 2-fold higher than those of cell lysates. The fold increase in protein amounts were 1.5-fold or comparable to those of CD63 and CD81 densities, respectively. The diameters of the particles of SNU719 and MKN74 were similar, suggesting that the change in protein content in each particle was negligible and that the increase in exosome numbers was 4- to 6-fold higher in SNU719 (Appendix A).

Table 2. Comparison of protein concentration and exosome size between EBVaGC and EBV-negative GC cell lines.

\begin{tabular}{ccccc}
\hline & PROTEIN $\left(\boldsymbol{\mu G} / \mathbf{1 0}^{5}\right.$ Cell) Fold & Cd63 Density Fold & CD81 Density Fold & Size \\
\hline Cell lysate & & & & \\
\hline SNU719/MKN7 & & 2.617 & 1.032 & \\
\hline SNU719/MKN74 & & 2.005 & 3.324 & \\
\hline Exosome & $2.29 / 0.515$ & & not applicable \\
\hline SNU719/MKN7 & 4.447 & 4.844 & 2.504 & $69.6 \mathrm{~nm} / 71.3 \mathrm{~nm}$ \\
\hline SNU719/MKN74 & $2.29 / 0.380$ & & & 0.976 \\
\hline
\end{tabular}

The meaning of each value is the same as Table 1. not applicable: electron microscopy was not performed for MK7.

\subsection{FACS Analysis of Human Monocyte-Derived Dendritic Cells Incubated with Gastric Cancer Exosomes}

Human monocyte-derived dendritic cells were cultured with exogenous exosomes of MKN74, MKN74 + EBV, and SNU719 (Figure 3A). Mature moDCs frequently formed cell aggregates, but such aggregates disappeared with the exosomes in MKN74 + EBV and SNU719. 


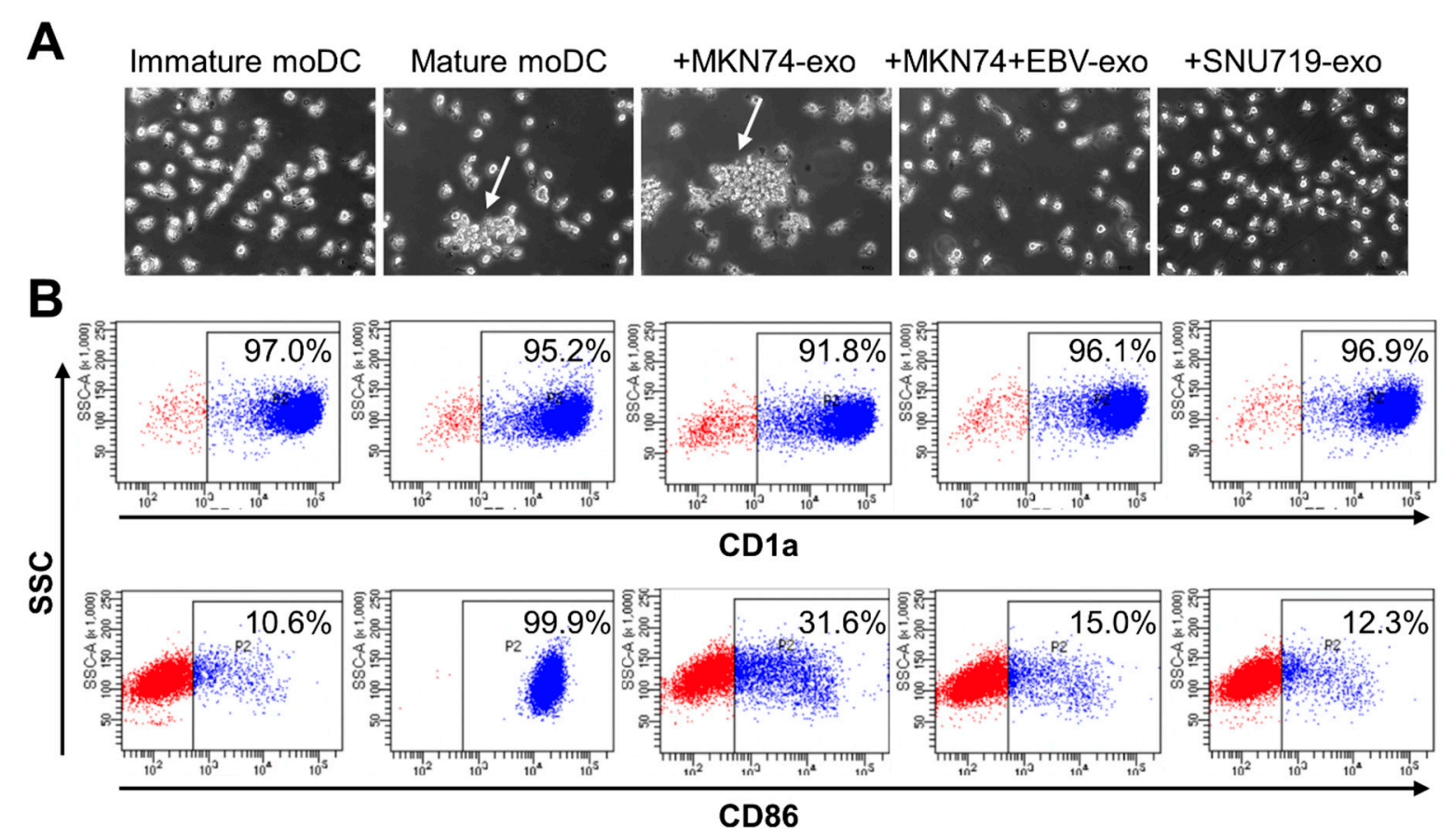

Figure 3. Differential effects of exosomes secreted by EBV-infected gastric cancer cells on the maturation phenotype of human monocyte-derived dendritic cells (moDCs). Immature DCs were either untreated or treated with the indicated exosomes or with LPS as a positive control for maturation. (A) Phase-contrast images of moDCs cultured with exosomes in EBV-infected cells. Large aggregates of cells (arrows) as reported for mature moDCs were found in MKN74-derived exome-treated moDCs but not in EBV-positive cell-derived exosome-treated cells. (B) Flow cytometry results of CD1a and CD86 on moDCs incubated with the indicated exosomes. The data are presented as $\%$ positive cells of indicated markers. The results are representative of three independent experiments from different donors.

Immature and mature markers were analyzed by FACS. When monocytes were stimulated with LPS, CD86-positive cells reached 99.9\% (data not shown), thereby confirming optimal experiment conditions. In a representative experiment, moDCs were shown to be CD1a-positive. The ratios of CD86 positive cells were $15.0 \%$ and $12.3 \%$ when incubated with the gastric cancer exosomes in MKN74 + EBV and SNU719, respectively. The ratios were significantly lower than those of MKN74 exosomes $(31.6 \%)$.

To exclude the possibility that a trace amount of EBV virus infected moDCs, we checked EBNA1-DNA in cultured monocytes, which were incubated with exosomes of SNU719. Using TaqMan quantitative PCR (qPCR) as described in the previous study, no amplicon was detected in 200 ng DNA of the exosome-treated monocyte. The limit of detection was $0.2 \mathrm{ng}$ of Namalawa cells, which harbor 1 copy per genome (two copies per cell) [17].

\subsection{Immunohistochemical Detection of Dendritic Cells in Gastric Cancer Tissue}

The clinicopathological parameters of gastric cancer cases used in this study are summarized in Table 3.

Dendritic cells expressing Langerin, CD1a, S100, CD83, CD86, and BDCA-2 were detected in gastric cancer tissue by immunohistochemical analysis (Figure 4). Dendritic cells expressing Langerin, CD1a, and S100 were identified as cells with abundant dendrites. Dendritic cells expressing BDCA-2, CD83, and CD86 were recognized as relatively small round cells. In EBVaGC, the dendrites of Langerin, CD1a, and S100 positive cells were more prominent compared to EBV-negative GC. Dendritic cells expressing Langerin, CD1a, S100, and BDCA-2 were homogeneously distributed in the tumors, although dendritic cells expressing CD83 and CD86 tended to aggregate in areas with abundant lymphocytes. 
Table 3. Clinicopathological characteristics of gastric cancer cases used for this study.

\begin{tabular}{ccc}
\hline Variables & EBV-Negative GC & EBVaGC \\
\cline { 2 - 3 } & $N=40$ & $N=41$ \\
\hline Age (y) & & $69(41-87)$ \\
Median (range) & $65(33-82)$ & $6(14.6 \%)$ \\
Sex & $7(17.5)$ & $35(85.4 \%)$ \\
Female & $33(82.5 \%)$ & $4.5(1.3-12.5)$ \\
Male & & \\
Size (cm) & $5.1(0.3-16.0)$ & $22(53.7 \%)$ \\
Median (range) & $5(12.5 \%)$ & $2(4.9 \%)$ \\
Location & $21(52.5 \%)$ & \\
Upper & $14(35.0 \%)$ & $20(48.8 \%)$ \\
Middle & & $21(51.2 \%)$ \\
Lower & $18(45.0 \%)$ & \\
Lauren classification & $22(55.0 \%)$ & $24(58.5 \%)$ \\
Diffuse & & $17(41.5 \%)$ \\
Intestinal & $21(52.5 \%)$ & \\
Tumor depth & $19(47.5 \%)$ &
\end{tabular}

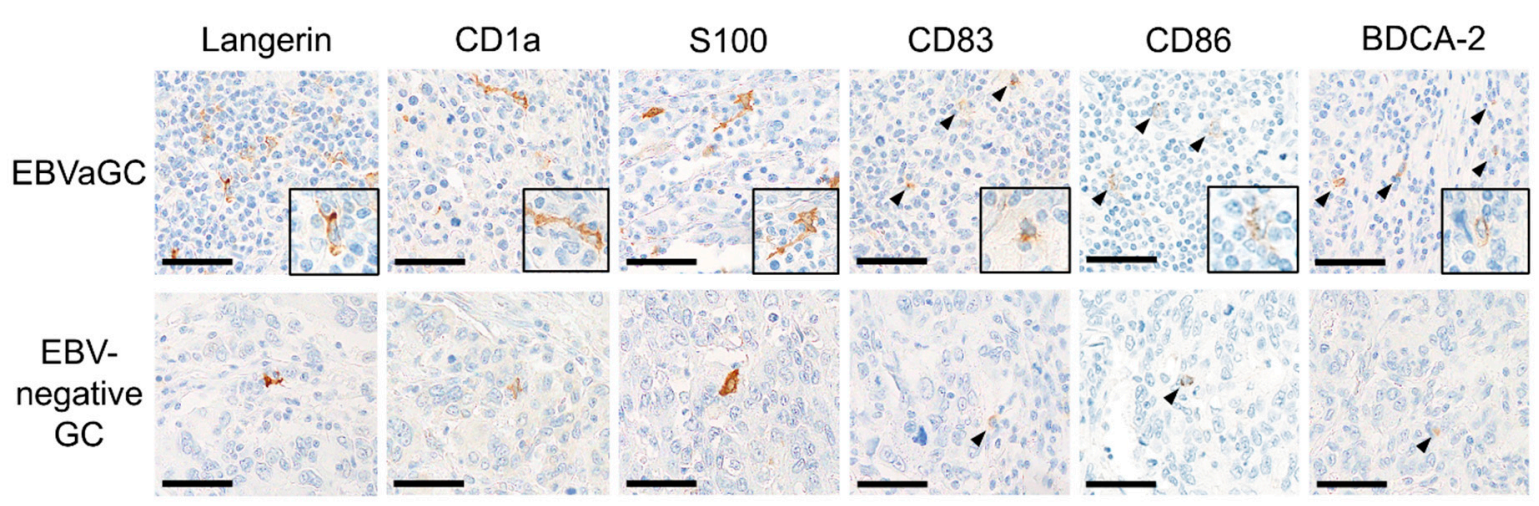

Figure 4. Representative figures of dendritic cells expressing each DC marker in gastric cancer tissue. DCs expressing Langerin, CD1a, and S100 were identified as cells with abundant dendrites. DCs expressing BDCA-2, CD83, and CD86 were identified as relatively small round cells (arrowhead). More abundant infiltration of dendritic cells in EBVaGC was observed than in EBV-negative gastric cancer for each DC marker. Immunohistochemical analysis of Langerin, CD1a, S100, CD83, CD86 and BDCA. Scale bar: $50 \mu \mathrm{m}$.

The number of dendritic cells per tumor area was significantly higher in EBVaGC cases than in EBV-negative GC cases for any of the DC markers (Figure 5, $p<0.001$ for Langerin, CD1a, CD83, CD86, and BDCA-2, and $p<0.05$ for S100, respectively, determined by using a Wilcoxon rank sum test).

\subsection{Correlation between Density of Dendritic Cells and Clinicopathological Factors}

The correlation between the density (number of dendritic cells per tumor area) and clinicopathological factors were evaluated among EBV-negative GC and EBVaGC; marked differences in both types of gastric carcinoma were observed (Table 4). In EBV-negative cases, the high density of DCs expressing CD1a was correlated with advanced (pT2-4) T stage, venous invasion, lymphatic invasion, and lymph node metastasis $(p<0.05)$. On the other hand, in EBVaGC, the high density of DCs expressing CD1a was correlated with the absence of lymphatic invasion $(p<0.05)$. The high density of DCs expressing CD83 was correlated with early (pT1) T stage $(p<0.05)$. Furthermore, the high density of DCs expressing BDCA-2 was correlated with diffuse histology and advanced (pT2-4) T stage $(p<0.05)$. 


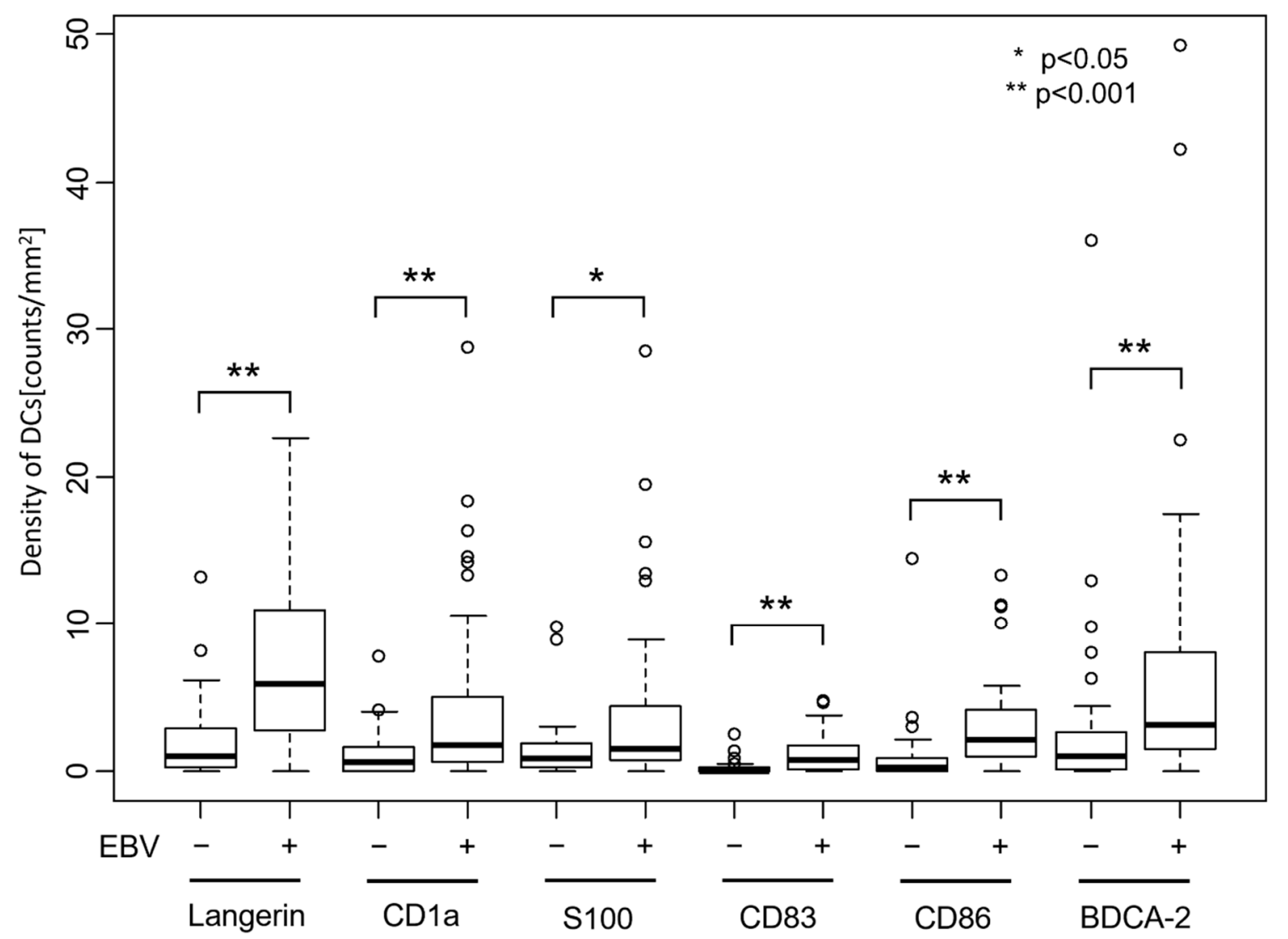

Figure 5. Number of dendritic cells that infiltrated gastric cancer tissue. Dendritic cells expressing each DC marker were counted, and the number per tumor area is shown. The number of DCs is significantly higher in EBVaGC than in EBV-negative gastric cancer for any of the DC markers ( $p<0.001$ for Langerin, CD1a, CD83, CD86, and BDCA-2. $p<0.05$ for S100, determined by using Wilcoxon rank sum test).

Table 4. Correlation between the number of dendritic cells per tumor area and clinicopathological factors.

\begin{tabular}{|c|c|c|c|c|c|c|c|c|c|c|c|c|c|}
\hline & & \multicolumn{6}{|c|}{ EBV-Negative GC } & \multicolumn{6}{|c|}{ EBVaGC } \\
\hline & & Langerin & CD1a & S100 & CD83 & CD86 & BDCA-2 & Langerin & CD1a & S100 & CD83 & CD86 & BDCA-2 \\
\hline \multirow{2}{*}{ Age } & $<65$ & 2.71 & 1.30 & 1.21 & 0.13 & 1.32 & 2.88 & 9.98 & 5.40 & 5.96 & 1.58 & 3.01 & 6.49 \\
\hline & $\geq 65$ & 1.16 & 1.09 & 1.69 & 0.27 & 0.49 & 2.64 & 7.10 & 4.56 & 3.29 & 0.90 & 3.29 & 7.71 \\
\hline \multirow{2}{*}{ Sex } & Male & 1.75 & 1.29 & 1.64 & 0.23 & 1.01 & 2.84 & 8.42 & 4.84 & 4.18 & 1.16 & $2.51 *$ & 6.25 \\
\hline & Female & 2.61 & 0.69 & 0.61 & 0.07 & 0.50 & 2.37 & 6.58 & 5.07 & 4.79 & 1.10 & $6.65 *$ & 13.19 \\
\hline Size & $<45 \mathrm{~mm}$ & 2.92 & 0.71 & 1.80 & 0.24 & 0.57 & 3.94 & 10.60 & 6.17 & 5.34 & 1.36 & 3.58 & 7.82 \\
\hline Lauren classification & Diffuse & 1.51 & 1.72 & 1.04 & 0.21 & 1.25 & 3.04 & 9.36 & 4.30 & 4.93 & 1.25 & 2.78 & $9.93^{*}$ \\
\hline \multirow{2}{*}{ Tumor depth } & Early & 1.92 & $0.50 *$ & 1.92 & 0.18 & 0.49 & 3.05 & 9.35 & 5.12 & 4.31 & $1.56^{*}$ & 3.37 & $5.45 *$ \\
\hline & Advanced & 1.88 & $1.94 *$ & 0.96 & 0.22 & 1.37 & 2.43 & 6.46 & 4.51 & 4.20 & $0.58^{*}$ & 2.90 & $9.82 *$ \\
\hline \multirow{2}{*}{ Venous invasion } & Absent & 2.08 & $0.54 *$ & 1.39 & 0.30 & 1.15 & 3.81 & 7.87 & 4.91 & 4.04 & 1.27 & 4.14 & 8.39 \\
\hline & Present & 1.68 & $1.97 *$ & 1.56 & 0.09 & 0.64 & 1.46 & 8.35 & 4.84 & 4.43 & 1.07 & 2.53 & 6.47 \\
\hline
\end{tabular}

Each value is the average number of dendritic cells per $\mathrm{mm}^{2}$ expressing the indicated DC marker. ${ }^{*} p<0.05$, determined by using Wilcoxon rank sum test. Significant correlations $(p<0.05)$ were identified.

For the comparison of the prognosis of EBV-negative GC and EBVaGC, gastric cancer cases were divided into DC-high and DC-low cases, and overall survival was compared using the Kaplan-Meier method (Figure 6). In EBVaGC, overall survival tended to be worse in cases with low CD83-positive DCs and CD86-positive DCs than in cases with high CD83-positive DCs and CD86-positive DCs, 
although the difference was not significant $(p=0.066$ and 0.26 , respectively, determined by using log-rank test). In EBV-negative GC, no significant difference in overall survival was observed between DC-high and DC-low cases (data not shown).
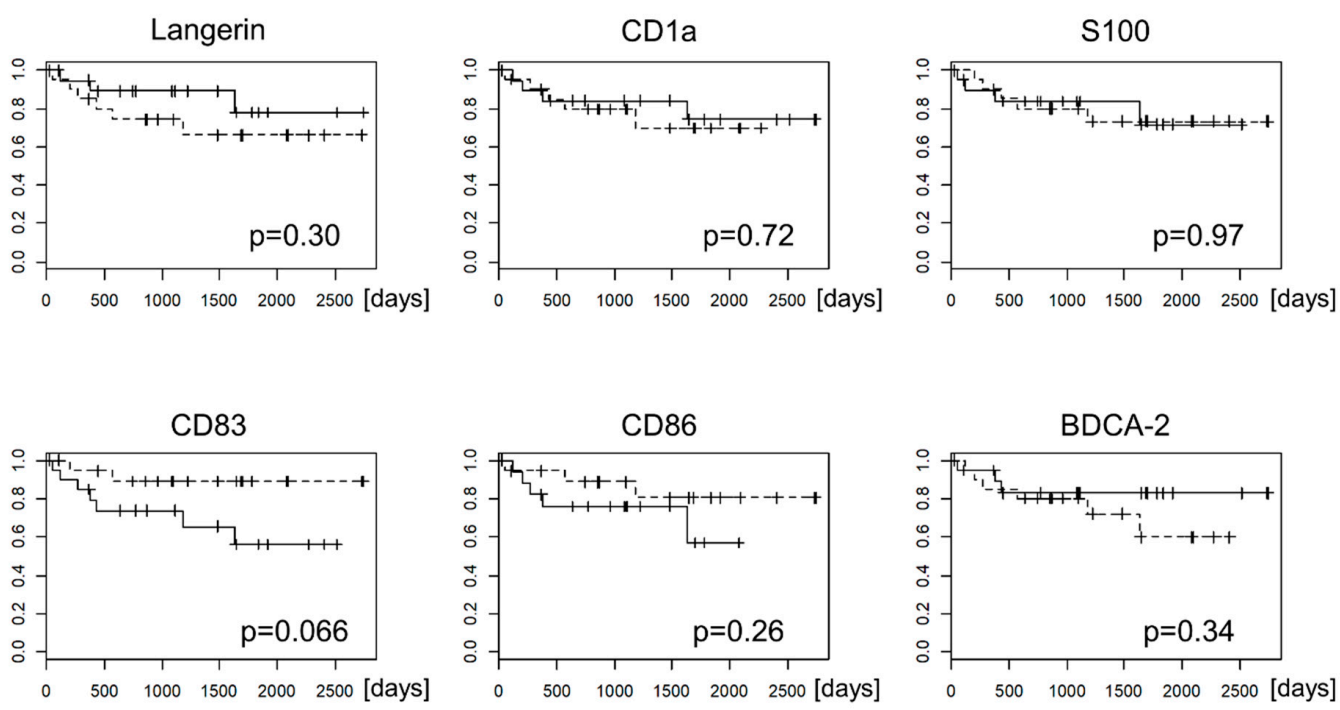

Figure 6. Kaplan-Meier curve of overall survival of patients with EBVaGC. Gastric cancer cases were divided into two groups according to the number of infiltrating DCs that express the indicated DC markers. The cases with less CD83-positive DCs tended to have worse prognosis, although this was not statistically significant $(p=0.06, \log$-rank test).

\subsection{Assessment of Mature DC Ratio in DC-High and DC-Low EBVaGC Cases}

The transition to mature dendritic cells in EBVaGC was assessed in detail, and CD83/CD1a ratio and CD86/CD1a ratios were determined to be significantly low in the group with abundant CD1a-positive dendritic cells (Figure 7A, $p<0.01$, determined by using Wilcoxon rank sum test). A similar result was also obtained in Langerin (Figure $7 \mathrm{~B}, p=0.08$ and $p=0.005$, respectively, determined by using Wilcoxon rank sum test).
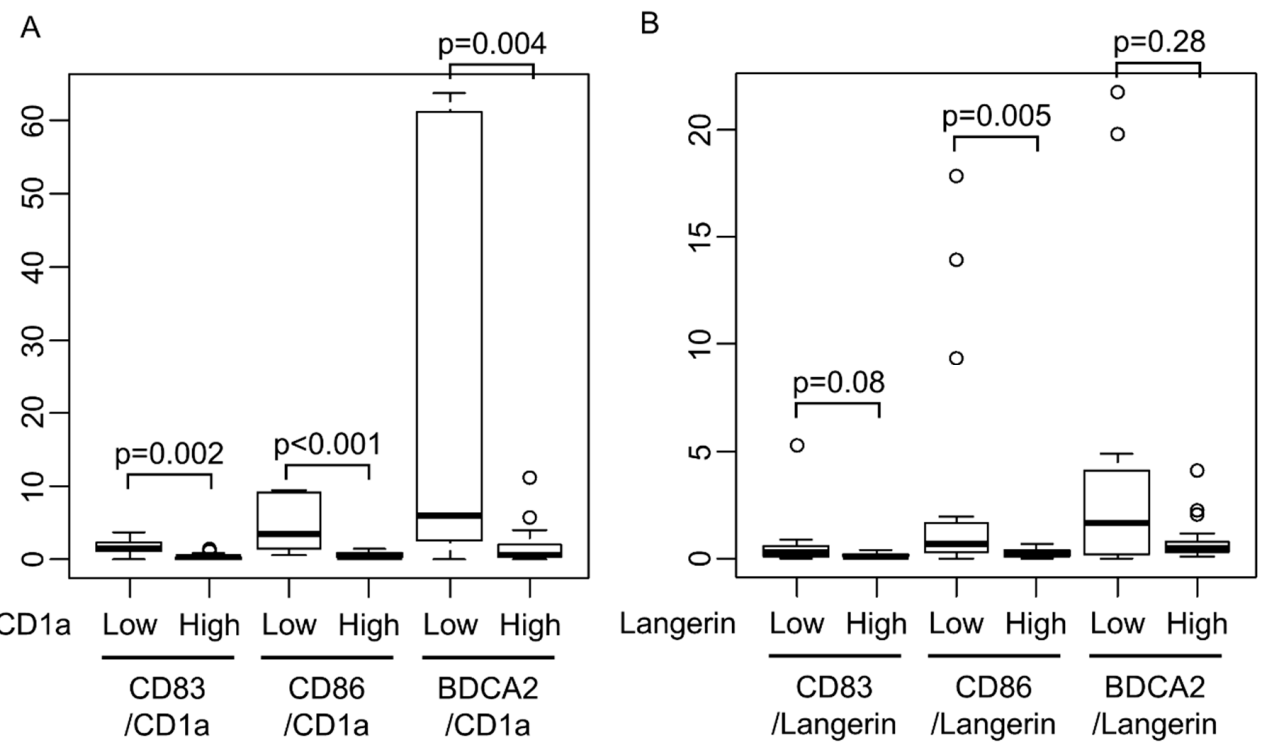

Figure 7. Mature DC ratio in DC-high and DC-low cases. CD83/CD1a ratio and CD86/CD1a ratios were significantly lower in CD1a-high cases than in CD1a-low cases $(\mathbf{A}, p<0.01$, determined by using Wilcoxon rank sum test). The similar result was also obtained in Langerin (B, $p=0.08$ and $p=0.005$, respectively, Wilcoxon rank sum test). 


\section{Discussion}

Much attention has been focused on exosomes as tools in intercellular communication that contribute to the establishment of the tumor microenvironment. It is becoming evident that EBV infection alters exosome contents, in addition to its capacity to incorporate virus-derived gene products [18]. However, little is known about the secretion and composition of exosomes from EBVaGC. As a first step in investigating EBVaGC exosomes, we analyzed exosomes from gastric cancer cell lines with and without artificial EBV-infection. We found a marked change in exosome marker proteins (CD63 and CD81) in both cellular production and exosome composition. An increase in the amount of CD63 and CD81 with a decrease in protein amount and particle size suggests that EBV infection considerably affects the dynamics of exosome secretion. Furthermore, in comparison with the amount of CD63 and CD81 proteins in EBV-negative gastric cancer cell lines, an increase in the amount of CD63 and CD81 protein in SNU719, which is an EBVaGC cell line, was observed, indicating an increase in exosome delivery to the microenvironment. Lio et al. [19] recently demonstrated that LMP1, a latent gene protein of EBV, promotes the secretion of exosomes in EBV-positive nasopharyngeal carcinoma. EBV may also control exosome secretion mechanisms in the infected cells of gastric carcinoma. It is worth noting that during lytic infection and egress of EBV, one of lytic gene products, BFRF1, recruits the endosomal sorting complex required for transport (ESCRT) to the nuclear envelope, which is involved in biogenesis of exosomes [20]. However, lytic infection process was not observed in EBV-infected gastric cancer cell lines and SNU719.

DCs are central to tumor immunity [4]. To prove that EBV-infected epithelial cells change the function of DCs through exosomes, flow cytometry analysis of moDCs was performed after culturing blood monocytes with gastric cancer-derived exosomes. CD86-positive moDC fraction was decreased in cultures with exosomes derived from MKN74 + EBV and SNU719 compared to that with exosomes derived from MKN74, indicating that EBV-infection imposes a negative effect on the induction of tumor immunity via their exosomes. Pegtel et al. [5] demonstrated that EBV-miRNAs in the exosomes from EBV-infected B cells are internalized by non-infected moDCs, resulting in the downregulation of immunoregulatory genes. Further studies are necessary to identify the constituents of EBVaGC exosomes, including EBV-miRNAs [18].

In the present study, we used dendritic cell markers suitable for immunohistochemical analysis to visualize tumor-associated dendritic cells (TA-DCs) in the tissue specimens. Langerin and CD1a were used as markers for immature moDCs and CD83 and CD86 for mature or activated DCs. S100 has been used as a general marker for DCs, and BDCA-2 is relatively specific to plasmacytoid DCs (pDCs) [11]. Here we show that the number of DCs was higher in EBVaGC than in EBV-negative GC with any of the markers. Our results are consistent with those in previous studies on EBVaGC $[7,8]$ and nasopharyngeal carcinoma [21,22]. Furthermore, we demonstrated that the significance of DCs is different between GC with and without EBV infection. As shown in Table 4, a significantly higher number of CD1a-positive DCs was observed in the clinical parameters of tumor invasion in EBV-negative GC, but such a correlation was not identified in EBVaGC. The number of CD1a-positive DCs was inversely higher in cases without lymphatic invasion. This shows the potential alteration of biological behavior in CD1a-positive DC, although further investigations are needed.

CD83-positive DCs were significantly lower in gastric carcinomas, showing deep infiltration. This result is in accordance with a report of van Beek et al. wherein CD83-positive dendritic cells were reportedly increased in EBVaGC in comparison to EBV-negative GC and among cases without lymph node metastasis [9]. As for the prognosis analysis, the cases with low numbers of CD83- and CD86-positive DCs showed a relatively worse prognosis than those with a high number of the same DCs. This result is concordant with that of Gong et al., who used fascin as a marker of mature dendritic cells [10], indicating that suppression of dendritic cells contributes to the progression of EBVaGC. Importantly, the proportion of CD83- and CD86-positive DCs to Langerin- or CD1a-positive DCs were significantly low in the group with a high number of Langerin- or CD1a-positive DCs. These findings indicate that there are some mechanisms that suppress the maturation of immature DCs in EBVaGC 
and correspond to the results of in vitro experiments on exosomes, i.e., a decrease in CD86-positive population by exosomes derived from EBV-positive gastric cancer cell lines.

The significance of BDCA-2-positive DCs is noteworthy. BDCA-2 is relatively specific to pDCs, whose origin is different from that of conventional DCs [23]. In the present study, a high number of BDCA-2-positive DCs was correlated with tumor invasion depth and was inversely proportional to the number of CD1a-positive cells in EBVaGC. However, the ratio of BDCA-2-positive DCs to immature DCs was similarly low in the group with a high number of Langerhans- and CD1a-positive DCs. Further studies are necessary to clearly elucidate the interrelationship among DC subtypes in EBVaGC.

Several limitations in the present study should be noted. We did not evaluate the profiles of exosome cargoes (especially viral proteins, non-coding RNAs, and microRNAs) and their relation to cellular counterparts. Specific subsets of EBV-lytic genes were consistently expressed in EBVaGC, although their function and their incorporation to the exosomes have not been clarified [24]. Furthermore, we focused on the tumor microenvironment, but EBV-exosome research encompasses the whole process of carcinogenesis, from the infection of epithelial cells to full-blown carcinoma. In this regard, Nanbo et al. demonstrated the contribution of exosomes in cell-to-cell viral transmission [25].

\section{Conclusions}

EBVaGC is a tumor with abundant DCs, including immature and mature moDCs and pDCs. Moreover, the maturation of DCs is suppressed, at least in part, by exosomes from EBV-infected epithelial cells.

Author Contributions: Conceptualization, M.F.; methodology, M.H. and A.K.; formal analysis, M.H. and A.K.; investigation, M.H., A.K., H.A., Y.M., and K.S.; resources, H.A., H.Y., Y.S., and T.U.; data curation, M.H.; writing-original draft preparation, M.H. and A.K.; writing - review and editing, M.F.; visualization, M.H. and A.K.; supervision, T.U. and M.F.; project administration, M.F.; funding acquisition, M.F. All authors have read and agreed to the published version of the manuscript.

Funding: This research was supported by Grants-in-Aid for Scientific Research (KAKENHI) from the Japan Society for the Promotion of Science (26253021 to M.F.).

Acknowledgments: We thank Kimiko Takeshita and Tomoko U. Irisa for their technical support, and Fumitaka Takeshita (National Cancer Center Research Institute, Tokyo, Japan) for helpful advice on exosome analysis.

Conflicts of Interest: The authors declare no conflict of interest. The funders had no role in the design of the study; in the collection, analyses, or interpretation of data; in the writing of the manuscript, or in the decision to publish the results.

\section{Appendix A Estimating the Number of Exosomes}

We assumed the following: (1) each exosome is spherical, (2) the total protein content of an exosome is proportional to the volume of the exosome, and (3) the protein content of CD63 and CD81 in an exosome is proportional to the surface area of the exosome. According to the results shown in Table 1 , the diameter of the exosome of MKN74 + EBV is approximately $1 / 2$ of the diameter of the exosome of MKN74, 1/4 the surface area 1/, and 1/8 the volume. The total amount of protein in the exosome sample corresponding to $10^{5}$ cells was approximately 0.8 times, and that of CD63 and CD81 was approximately 2.8 times. Therefore, we estimated that the number of exosomes is approximately $6.4(=0.8 \times 8)$ to $11.2(=2.8 \times 4)$ times. In the results shown in Table 2, the diameters of the exosomes in SNU719 and MKN74 were almost equal, but the amount of total protein amount and that of CD63 and CD81 were different by 6-8-fold. Therefore, we estimated the number of exosomes to be different by 6-8-fold.

\section{References}

1. Young, L.S.; Rickinson, A.B. Epstein-Barr virus: 40 years on. Nat. Rev. Cancer 2004, 4, 757-768. [CrossRef]

2. Fukayama, M.; Abe, H.; Kunita, A.; Shinozaki-Ushiku, A.; Matsusaka, K.; Ushiku, T.; Kaneda, A. Thirty years of Epstein-Barr virus-associated gastric carcinoma. Virchows Arch. 2020, 476, 353-365. [CrossRef] 
3. Sano, T.; Coit, D.G.; Kim, H.H.; Roviello, F.; Kassab, P.; Wittekind, C.; Yamamoto, Y.; Ohashi, Y. Proposal of a new stage grouping of gastric cancer for TNM classification: International Gastric Cancer Association staging project. Gastric Cancer 2017, 20, 217-225. [CrossRef] [PubMed]

4. Balkwill, F.R.; Capasso, M.; Hagemann, T. The tumor microenvironment at a glance. J. Cell Sci. 2012, 125, 5591-5596. [CrossRef] [PubMed]

5. Zhang, L.; Yu, D. Exosomes in cancer development, metastasis, and immunity. Biochim. Biophys. Acta Rev. Cancer 2019, 1871, 455-468. [CrossRef] [PubMed]

6. Pegtel, M.D.; Cosmopoulos, K.; Thorley-Lawson, D.A.; van Eijndhoven, M.A.; Hopmans, E.S.; Lindenberg, J.L.; de Gruijl, T.D.; Würdinger, T.; Middeldorp, J.M. Functional delivery of viral miRNAs via exosomes. Proc. Natl. Acad. Sci. USA 2010, 107, 6328-6333. [CrossRef] [PubMed]

7. Saiki, Y.; Ohtani, H.; Naito, Y.; Miyazawa, M.; Nagura, H. Immunophenotypic characterization of Epstein-Barr virus-associated gastric carcinoma: Massive infiltration by proliferating CD8+ T-lymphocytes. Lab. Invest. 1996, 75, 67-76. [PubMed]

8. Kijima, Y.; Ishigami, S.; Hokita, S.; Koriyama, C.; Akiba, S.; Eizuru, Y.; Aikou, T. The comparison of the prognosis between Epstein-Barr virus (EBV)-positive gastric carcinomas and EBV-negative ones. Cancer Lett. 2003, 200, 33-40. [CrossRef]

9. van Beek, J.; zur Hausen, A.; Snel, S.N.; Berkhof, J.; Kranenbarg, E.; van de Velde, C.J.; van den Brule, A.J.; Middeldorp, J.M.; Meijer, C.J.; Bloemena, E. Morphological evidence of an activated cytotoxic T-cell infiltrate in EBV-positive gastric carcinoma preventing lymph node metastases. Am. J. Surg. Pathol. 2006, 30, 59-65. [CrossRef] [PubMed]

10. Gong, L.P.; Chen, J.N.; Xiao, L.; He, Q.; Feng, Z.Y.; Zhang, Z.G.; Liu, J.P.; Wei, H.B.; Shao, C.K. The implication of tumor-infiltrating lymphocytes in Epstein-Barr virus-associated gastric carcinoma. Hum. Pathol. 2019, 85, 82-91. [CrossRef] [PubMed]

11. Hubert, M.; Gobbini, E.; Bendriss-Vermare, N.; Caux, C.; Valladeau-Guilemond, J. Human tumor-infiltrating dendritic cells: From in situ visualization to high-dimensional analyses. Cancers 2019, 11, 1082. [CrossRef] [PubMed]

12. Surówka, J.; Wertel, I.; Okła, K.; Bednarek, W.; Tarkowski, R.; Kotarski, J. Influence of ovarian cancer type I and type II microenvironment on the phenotype and function of monocyte-derived dendritic cells. Clin. Transl. Oncol. 2017, 19, 1489-1497. [CrossRef] [PubMed]

13. Imai, S.; Nishikawa, J.; Takada, K. Cell-to-cell contact as an efficient mode of Epstein-Barr virus infection of diverse human epithelial cells. J. Virol. 1998, 72, 4371-4378. [CrossRef] [PubMed]

14. Yoshioka, Y.; Konishi, Y.; Kosaka, N.; Katsuda, T.; Kato, T.; Ochiya, T. Comparative marker analysis of extracellular vesicles in different human cancer types. J. Extracell. Vesicles 2013, 18, 2. [CrossRef]

15. Théry, C.; Witwer, K.W.; Aikawa, E.; Alcaraz, M.J.; Anderson, J.D.; Andriantsitohaina, R.; Antoniou, A.; Arab, T.; Archer, F.; Atkin-Smith, G.K.; et al. Minimal information for studies of extracellular vesicles 2018 (MISEV2018): A position statement of the International Society for Extracellular Vesicles and update of the MISEV2014 guidelines. J. Extracell. Vesicles 2018, 7, 1535750. [CrossRef]

16. R Core Team. R: A Language and Environment for Statistical Computing; R Foundation for Statistical Computing: Vienna, Austria, 2018; Available online: https://www.R-project.org/ (accessed on 25 September 2020).

17. Nakayama, A.; Abe, H.; Kunita, A.; Saito, R.; Kanda, T.; Yamashita, H.; Seto, Y.; Ishikawa, S.; Fukayama, M. Viral loads correlate with upregulation of PD-L1 and worse patient prognosis in Epstein-Barr Virus-associated gastric carcinoma. PLoS ONE 2019, 14, e0211358. [CrossRef]

18. Zhao, M.; Nanbo, A.; Sun, L.; Lin, Z. Extracellular vesicles in Epstein-Barr virus' life cycle and pathogenesis. Microorganisms 2019, 7, 48. [CrossRef]

19. Liao, C.; Zhou, Q.; Zhang, Z.; Wu, X.; Zhou, Z.; Li, B.; Peng, J.; Shen, L.; Li, D.; Luo, X.; et al. Epstein-Barr virus-encoded latent membrane protein 1 promotes extracellular vesicle secretion through syndecan-2 and synaptotagmin-like-4 in nasopharyngeal carcinoma cells. Cancer Sci. 2020, 111, 857-868. [CrossRef]

20. Lee, C.P.; Liu, P.T.; Kung, H.N.; Su, M.T.; Chua, H.H.; Chang, Y.H.; Chang, C.W.; Tsai, C.H.; Liu, F.T.; Chen, M.R. The ESCRT machinery is recruited by the viral BFRF1 protein to the nucleus-associated membrane for the maturation of Epstein-Barr Virus. PLoS Pathog. 2012, 8, e1002904. [CrossRef] 
21. Braz-Silva, P.H.; Vitale, S.; Butori, C.; Guevara, N.; Santini, J.; Magalhães, M.; Hofman, P.; Doglio, A. Specific infiltration of langerin-positive dendritic cells in EBV-infected tonsil, Hodgkin lymphoma and nasopharyngeal carcinoma. Int. J. Cancer 2011, 128, 2501-2508. [CrossRef]

22. Nilsson, J.S.; Abolhalaj, M.; Lundberg, K.; Lindstedt, M.; Greiff, L. Dendritic cell subpopulations in nasopharyngeal cancer. Oncol. Lett. 2019, 17, 2557-2561. [CrossRef]

23. Manz, M.G. Plasmacytoid dendritic cells: Origin matters. Nat. Immunol. 2018, 19, 652-654. [CrossRef] [PubMed]

24. Borozan, I.; Zapatka, M.; Frappier, L.; Ferretti, V. Analysis of Epstein-Barr Virus Genomes and Expression Profiles in Gastric Adenocarcinoma. J. Virol. 2018, 92, e01239-17. [CrossRef] [PubMed]

25. Nanbo, A.; Ohashi, M.; Yoshiyama, H.; Ohba, Y. The Role of Transforming Growth Factor $\beta$ in Cell-to-Cell Contact-Mediated Epstein-Barr Virus Transmission. Front Microbiol. 2018, 9, 984. [CrossRef] [PubMed]

Publisher's Note: MDPI stays neutral with regard to jurisdictional claims in published maps and institutional affiliations.

(C) 2020 by the authors. Licensee MDPI, Basel, Switzerland. This article is an open access article distributed under the terms and conditions of the Creative Commons Attribution (CC BY) license (http://creativecommons.org/licenses/by/4.0/). 